\title{
Modificación de un material didáctico interactivo en la búsqueda de un aprendizaje más adaptativo \\ Modification of an interactive didactic material in search of a more adaptive learning
}

\author{
Hernández-Castellano, Pedro M. ${ }^{1}$, Araña-Suárez, Roberto E. ${ }^{1}$, Marrero-Alemán, María Dolores ${ }^{1}$, \\ Sánchez-Morales, Carlos J. ${ }^{1}$ \\ pedro.hernandez@ulpgc.es, roberto.arana101@alu.ulpgc.es,mariadolores.marrero@ulpgc.es, \\ carlos.sanchez@ulpgc.es
}

${ }^{1}$ Grupo de Innovación Educativa Ingeniería de Fabricación

Universidad de Las Palmas de Gran Canaria

Las Palmas de Gran Canaria, España

\begin{abstract}
Resumen- Este trabajo presenta la evolución de un material didáctico interactivo orientado hacia el sistema de especificaciones geométricas de productos, ISO GPS. El grupo de Innovación Educativa Ingeniería de Fabricación de la Universidad de Las Palmas de Gran Canaria ha buscado aportar un grado de adaptatividad más alto a este material implementándolo directamente en la plataforma Moodle. Se han reorganizado los contenidos en varios bloques para mejorar su progresividad, siendo necesario superar cuestionarios de conceptos básicos para ir avanzando hacia nuevos contenidos. También se le han incorporado nuevos recursos como videos interactivos de resolución de problemas y un simulador gráfico para la visualización de las tolerancias y ajustes. Se ha empleado metodologías con la participación activa y colaborativa de estudiantes, tanto para el desarrollo como en la evaluación de este nuevo material didáctico. Los resultados obtenidos evidencian que las mejoras introducidas han tenido un impacto positivo en el aprendizaje en los estudiantes, y se ha mostrado también como una herramienta muy adecuada para su uso en una modalidad de educación híbrida que integre la docencia presencial y la telemática.
\end{abstract}

Palabras clave: Aprendizaje adaptativo, Prácticas educativas en abierto, Material didáctico interactivo

Abstract- This work presents the evolution of an interactive teaching material oriented towards the system of geometric product specifications, ISO GPS. The Educational Innovation Group Ingeniería de Fabricación of the University of Las Palmas de Gran Canaria has sought to provide a higher level of adaptivity to this material by implementing it directly on the Moodle platform. The contents have been reorganized into several blocks to improve their progressivity, making it necessary to pass basic concepts questionnaires to progress towards new contents. New resources have also been incorporated, such as interactive problem-solving videos and a graphic simulator for displaying the tolerances. Methodologies have been used with the active and collaborative participation of students, both for the development and evaluation of this new teaching material. The results obtained show that the improvements introduced have had a positive impact on student learning and it has also been shown to be a very suitable tool for use in a hybrid education modality in which face-to-face teaching and telematics are integrated.
Keywords: Adaptive learning, Open educational practices, Interactive didactic material.

\section{INTRODUCCIÓN}

El empleo del aprendizaje adaptativo en el ámbito universitario ha experimentado un importante auge en los últimos años, según se recoge en las numerosas referencias publicadas en el informe Horizon 2017 Edición Educación Superior (Becker et al., 2017). Se debe tener en cuenta que esta tipología de aprendizaje forma parte del concepto más amplio de personalización de la enseñanza, basada en un modelo educativo centrado en el aprendizaje (Lerís \& Sein-Echaluce, 2011). Si bien existe desde hace algún tiempo una amplia coincidencia en la necesidad de implantar en todos los contextos educativos las metodologías de aprendizaje personalizado, no ha sido hasta la última década cuando se han potenciado una gran cantidad de herramientas o plataformas tecnológicas (Graduate XXI, 2018), que han facilitado la incorporación de estos nuevos modelos de aprendizaje.

Aunque existen varias categorías de aprendizaje adaptativo, se pueden distinguir dos modelos generales (EduTrends, 2014): el impulsado por el contenido y el impulsado por la evaluación. El primer caso se basa en el análisis de los datos obtenidos tras la interacción de los estudiantes con los contenidos y es el docente el que decide lo que se debe modificar en el contenido para favorecer el aprendizaje personalizado. El segundo caso es el que se identifica más directamente con el aprendizaje adaptativo. En él es una herramienta informática la que analiza y realiza los ajustes necesarios en función de los resultados de evaluación del estudiante.

Si bien es cierto que una adecuada implantación de estas técnicas requiere que, en el ámbito universitario, se modifiquen algunos aspectos que priman el grupo frente al estudiante. Es el caso, por ejemplo, de la tasa de éxito, indicador de la eficiencia del aprendizaje en el modelo universitario actual, que hace referencia a la relación entre el número total de créditos superados por el grupo y el número total de créditos presentados a examen, también del grupo. No obstante, en un artículo sobre 
aprendizaje adaptativo en Moodle los autores demostraron que la aplicación de técnicas de aprendizaje adaptativo permitió aumentar la tasa de éxito de las titulaciones, al mejorar la motivación de los estudiantes a los que se les ha adaptado su plan docente en función de sus debilidades y fortalezas (López et al., 2015).

\section{CONTEXTO}

Este trabajo ha surgido como una evolución de un material didáctico interactivo sobre el sistema ISO GPS, Especificaciones Geométricas del Producto. Este material didáctico permitió resolver con éxito unas dificultades de aprendizaje generalizadas que se habían identificado en la asimilación y aplicación práctica de este bloque de contenidos. Fue un primer paso en la introducción de cierto grado de adaptatividad al aprendizaje, incorporando elementos de navegación e interacción que permitían que el estudiante pudiera usarlo de formas variadas y adaptadas a su estilo y ritmo de aprendizaje.

Este material ha presentado buenos resultados en su aplicación durante los últimos cursos académicos (Pedro Manuel Hernández-Castellano et al., 2019). Se ha observado una notable mejora en el proceso de enseñanza aprendizaje, con una mejor integración de los contenidos teóricos y prácticos, y propiciando una actitud más activa y participativa en los estudiantes. Aún con ello, se ha analizado el uso de este material didáctico, identificando varios aspectos que podían mejorar significativamente el grado de adaptación al estudiante. Éste ha sido el objetivo fundamental del presente trabajo, al que se ha sumado el de incorporar contenidos adicionales complementarios. Otro objetivo del trabajo ha sido el de realizar una nueva estructuración y organización del conjunto de los contenidos para introducir elementos de autoevaluación. También se ha buscado integrar nuevos recursos didácticos que pudieran mejorar la experiencia de aprendizaje del estudiante.

Este material didáctico está siendo utilizado principalmente en asignaturas de introducción a la Ingeniería de los Procesos de Fabricación que están recogidas en todos los títulos de grado en Ingeniería del ámbito industrial. La mayoría de estas asignaturas se encuentran en segundo curso y cuentan con 4,5 ECTS, con lo que no es posible dedicar muchas sesiones presenciales a ninguno de los bloques temáticos en los que están estructuradas. La mayoría de los estudiantes usan este material didáctico al inicio del tercer semestre, cuando han cursado unas pocas asignaturas del bloque específico del título. A muchos les resulta difícil visualizar y entender el concepto de tolerancia dimensional porque la aplicación práctica de este concepto todavía no la han visto en ninguna asignatura. Solo en la titulación de Ingeniería en Diseño Industrial y Desarrollo de Productos, se imparten estos contenidos en la asignatura de Procesos Industriales en el sexto semestre. Los estudiantes de esta titulación han tenido la oportunidad de cursar muchas más asignaturas específicas del título, en alguna de las cuales este concepto se ha sido introducido y relacionado con otros afines. Este material didáctico debe permitir la adaptación a estudiantes con estas diferencias, y además, a los diferentes estilos y ritmos de aprendizaje que estos pueden presentar.

\section{DESCRIPCIÓN}

El material didáctico objeto de este trabajo surgió dentro de un proyecto de renovación pedagógica de un grupo de innovación educativa que implicó desde un primer momento a estudiantes motivados por esta temática a través de las asignaturas de Prácticas Externas y Trabajo fin de Grado. Las primeras experiencias fueron muy positivas y superaron las expectativas iniciales, lo que permitió abrir una nueva línea de trabajo en este grupo (Pedro M Hernández-Castellano et al., 2017). En los siguientes apartados se describen las metodologías aplicadas en el desarrollo de este trabajo, así como el material didáctico de partida. A continuación, se describen brevemente las tecnologías concretas aplicadas en el desarrollo del nuevo material didáctico, y los nuevos recursos didácticos que se han incorporado al mismo.

\section{A. Metodologías}

La metodología de trabajo en la que los estudiantes se implican como socios comprometidos y valiosos en procesos de enseñanza y aprendizaje es denominada Students as Partners (SaP). Esta metodología encaja perfectamente dentro de las denominadas Prácticas Educativas en Abierto (Open Education Practices, OEP) que buscan nuevos modelos que promuevan la participación, colaboración y apertura a los diferentes agentes del entorno educativo para generar experiencias de gran valor. Estas prácticas propician la interacción e implicación de los estudiantes, con actividades sencillas, flexibles y colaborativas. Se fomenta la creatividad, los procesos de co-creación y el trabajo en equipo, para conseguir un aprendizaje más significativo entre iguales y con ello un empoderamiento del estudiante (Cronin \& Maclaren, 2018).

La metodología SaP reconoce que estudiantes y profesores tienes diferentes puntos de vista sobre un tema y ambos son complementarios. Estas prácticas en la que docentes y estudiantes trabajan juntos trata, sobre todo, de compartir responsabilidades e ideas a través del diálogo y la colaboración entre ambas partes, permitiendo generar mejoras sustanciales tanto en el proceso de enseñanza como enriqueciendo significativamente el proceso de aprendizaje (Matthews et al., 2019). El uso de esta metodología aumenta la motivación, implicación y compromiso del estudiante, ya que este se enfrenta a un problema desafiando los roles en los que normalmente se encuentran el profesorado y el alumnado, pasando a ser ambos compañeros con un fin común. Esta metodología no favorece solo a los estudiantes, los docentes al trabajar con ellos obtienen una mayor conciencia del impacto que tienen sus actividades y una mayor capacidad de analizar los objetivos que se habían planteado. Además, comprenden mejor lo que sucede en el aula y lo que pasa por la mente de las personas que participan en esas actividades académicas (Bovill \& Felten, 2016).

Por otro lado, la metodología de enseñanza invertida (Flipped Teaching) se aplica básicamente para que los contenidos sean estudiados previamente a las sesiones presenciales en el aula, que se orientan a la consolidación de esos conocimientos mediante la reflexión grupal y la realización de actividades complementarias (García-Peñalvo et al., 2019). Se pueden utilizar múltiples tipos de recursos educativos para facilitar el aprendizaje del estudiante en esa fase previa de trabajo autónomo, que puede ser tanto individual como cooperativo. Se trata de una metodología que bien aplicada permite conseguir una participación más activa del estudiante, logrando una mayor eficiencia del trabajo, tanto en el aula como fuera de ella, y enriqueciendo la experiencia de aprendizaje. Lo más habitual es recurrir a videos descriptivos de los contenidos a trabajar, y es mucho menos frecuente usar materiales 
didácticos específicamente diseñados para ser usados con esta metodología y que a su vez integren varios tipos de recursos (Enfield, 2016).

\section{B. Material didáctico de partida}

El material didáctico de referencia que se quería modificar consiste en un documento interactivo en formato PDF que incluye gran cantidad y variedad de elementos multimedia para motivar al estudiante y orientarlo en su proceso de aprendizaje (Hernández-Castellano Pedro M. et al., 2017).

Este trabajo se comenzó realizando un análisis detallado de material de partida para clasificar y reordenar sus contenidos. Como resultado de este análisis se concluyó que seguían existiendo numerosos conceptos teóricos muy similares y fáciles de confundir, cuyas definiciones no se identificaban con facilidad. Algunos subapartados presentaban dificultades para identificar con claridad su inicio y finalización, y algunas representaciones gráficas muestran dificultades de interpretación. El material recoge algunos ejercicios guiados que requieren más claridad para entender fácilmente las soluciones adoptadas. Algunos recursos multimedia incorporados al documento PDF han de dejado de funcionar por motivos técnicos.

También se vio necesario introducir los conceptos de tolerancias geométricas y de acabado superficial para tener una mayor visión de conjunto de las especificaciones geométricas de productos. Y se había propuesto ya en un trabajo previo relacionado con este material didáctico, que sería de gran interés contar con un módulo gráfico interactivo que el estudiante pudiera usar para visualizar y simular las tolerancias y ajustes que estuviera estudiando.

\section{Tecnologías}

Para la mejora en la adaptatividad del aprendizaje que se quería aportar a este material didáctico, se vio necesario trasladar los contenidos del mismo a un sistema de gestión de contenidos de aprendizaje (LCMS) para aprovechar las herramientas que estos sistemas contemplan. Para su implementación se usó Moodle, versión 3.9, como plataforma institucional, que permitía el desarrollo de entornos de aprendizaje personalizado, y se realizó un detallado análisis de todas las herramientas que ofrecía.

De todas ellas se identificó que las actividades estructuradas H5P y su amplia biblioteca de recursos, permitía cubrir las necesidades a la vez que incluir muchos elementos de interacción con el estudiante. Además, esta nueva versión de Moodle contempla una integración total de esta potente herramienta y permite su almacenamiento en la propia plataforma. También, se identificó que la herramienta GeoGebra permitiría desarrollar una aplicación para implementar un módulo gráfico interactivo de simulación y comparación de tolerancias y ajustes, que se integraría directamente en la plataforma.

\section{Nuevo material didáctico}

Este nuevo material didáctico se ha estructurado en 5 bloques de contenidos que de forma progresiva va presentando a los estudiantes los conceptos requeridos. Un primer bloque con un único capítulo de introducción a la normalización y la necesidad de establecer un sistema normalizado de Tolerancias y Ajustes. Un segundo bloque con 3 capítulos descriptivos de los diferentes tipos de tolerancias: dimensionales, geométricas y de acabado superficial. Un tercer bloque con un solo capítulo donde se exponen los conceptos básicos del sistema ISO GPS para las tolerancias dimensionales y los ajustes. Un cuarto bloque con un capítulo de conceptos avanzados, y que da acceso a un documento que recoge las tablas de la norma de aplicación, y a un video explicativo de su uso. En este cuarto bloque también se puede acceder al simulador gráfico de tolerancias y ajustes, desarrollado a través de GeoGebra, para que puedan poner en práctica los conceptos adquiridos, figura 1 .

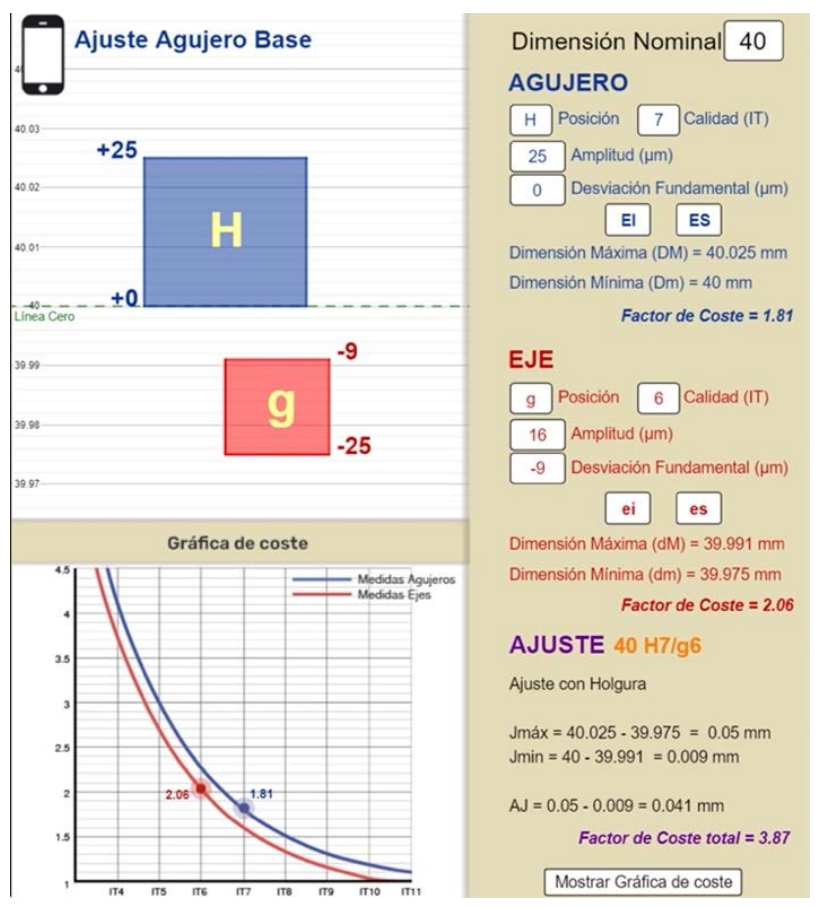

Figura 1. Simulador gráfico interactivo

El quinto y último bloque es de aplicación práctica a ejercicios guiados y problemas reales con múltiples soluciones. En los ejercicios guiados se expone la resolución paso a paso, mientras que para explicar como se resuelve un problema de tipo inverso se ha desarrollado un recurso de video interactivo con varios puntos de control, figura 2. En estos puntos el estudiante tiene que tomar decisiones que afectan a la resolución del ejercicio y conducen a diferentes soluciones viables. Una vez completada cada una de estas alternativas se puede acceder a las otras, para entender las diferencias entre ellas. Al final de este recurso multimedia se comparan todas las alternativas entre sí, tanto desde el punto de vista técnico como económico, figura 3.

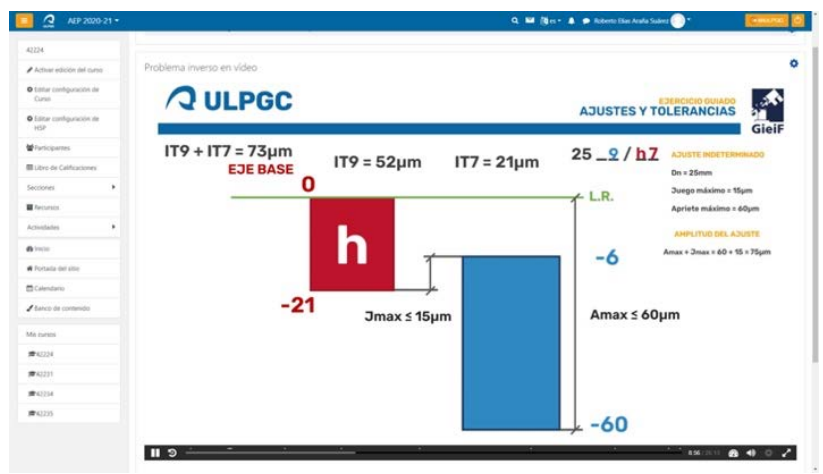

Figura 2. Imagen del video interactivo. 




Figura 3. Flujo de opciones del video interactivo

Todos los capítulos y la mayoría de los recursos mencionadas se han desarrollado a partir de actividades H5P. A los diferentes módulos se acceden de forma secuencial a través de unos cuestionarios que permitan evidenciar que ha alcanzado un nivel de dominio mínimo en los conceptos abordados. Estos cuestionarios tienen un número máximo de 3 intentos y se generan de forma aleatoria a partir de un amplio banco de preguntas tipo que a su vez presentan diversas variantes tanto en su enunciado como en las respuestas posibles. La calificación mínima requerida para superar estos cuestionarios es de 6 puntos sobre 10. Estos cuestionarios permiten una retroalimentación específica para cada pregunta tipo, con la que se orienta al estudiante sobre los aspectos que debe reforzar antes de volver a intentarlo. Una vez superados los diferentes módulos el estudiante puede acceder a cualquiera de ellos para reforzar los conocimientos adquiridos.

\section{Resultados}

Este nuevo material didáctico se ha usado de forma piloto en el primer semestre del curso 2020/21 en la asignatura de Procesos Industriales del Grado en Ingeniería en Diseño Industrial y Desarrollo de Productos. En esta asignatura se cuenta con mucha información que ha permitido evaluar el impacto del material didáctico previo y por tanto, se tomará esta información de referencia para evaluar el primer impacto en la introducción del nuevo material didáctico. Esta asignatura cuenta con 46 estudiantes matriculados.

Se ha hecho partícipe al alumnado de esta asignatura de un proceso de evaluación del nuevo material didáctico. Se ha habilitado un foro de mejora del material didáctico en el que podían ir aportando incidencias y sugerencias a medida que tenían acceso a los diferentes módulos. Esta información permitió ir introduciendo varias modificaciones durante las semanas previas a la prueba de evaluación de estos contenidos en la asignatura.

Una vez completada esa prueba de evaluación parcial, también se les ha solicitado a los estudiantes que participaran en otro foro de valoración general del material didáctico.
Debían aportar sus impresiones relativas a: la facilidad de uso y navegación, de los recursos gráficos y multimedia, de las dificultades de aprendizaje encontradas, la estimación de horas dedicadas a su estudio, y cualquier otra información que consideraran de interés. En esta actividad participaron 18 estudiantes lo que supone un $40 \%$ de los matriculados y casi el $50 \%$ de los que se habían presentado a la prueba de evaluación parcial. La opinión general manifestada por casi todos los participantes fue que el material resultó muy intuitivo y cómodo de usar. El material gráfico, los videos y el simulador facilitaron el aprendizaje de esos contenidos. El tiempo de dedicación por bloque estaba entre 1 y 2 horas, y se requeriría más tiempo con materiales tradicionales. Seguían siendo necesarias las sesiones presenciales de prácticas de aula donde se aclaran y justifican determinados aspectos más específicos. Los aspectos a mejorar se centraban principalmente en la configuración y retroalimentación de los test, y en algún aspecto puntual de la navegación o resolución de algún bloque.

La evaluación de estos contenidos se realiza a través de un test de conceptos que incluye unos sencillos ejercicios de aplicación, y de un problema más elaborado donde han de justificar la solución alcanzada entre varias posibles. En los cursos 2019/20 y 2020/21 estas dos pruebas se han pasado a modalidad telemática y en formato libro abierto en el que los estudiantes pueden usar todo el material que requieran. Tienen un tiempo limitado de 20 minutos para completar el test, que se genera de forma aleatoria para cada estudiante y se ha de responder de forma secuencial. Para el problema se realiza en un cuestionario independiente con un enunciado con datos generados también de forma aleatoria, y para el que disponen de 60 minutos para introducir en una pregunta de tipo ensayo la respuesta que proponen y acompañarla de un archivo con la resolución justificada del mismo. Se ha intentado mantener la mayor similitud posible en la prueba de evaluación respecto a la que se venía realizando a la modalidad presencial en cursos anteriores. Esta prueba se hace de forma parcial a mitad del semestre y se repite en cada una de las convocatorias oficiales para los estudiantes que no hayan podido superarla previamente.

Los resultados obtenidos en la prueba de evaluación parcial para los últimos cursos académicos en los que se ha usado el material didáctico previo se muestran en la tabla 1. Esta información permite evaluar el primer impacto de la introducción de este nuevo material didáctico. De estos datos se puede observar que el curso pasado 19/20, donde se utilizó el material didáctico anterior, aumentaron significativamente los porcentajes de estudiantes que aprobaron o mostraron un alto potencial para aprobar, con una calificación superior a 4, tanto en el test de conceptos como en el problema. A pesar de las excepcionales condiciones que se produjeron en el segundo semestre del curso pasado, estos buenos resultados se achacan al alto potencial de trabajo autónomo que ya ofrecía el material didáctico anterior. En este último curso 20/21 se puede destacar un significativo aumento de la calificación media de los estudiantes aprobados respecto al curso pasado 19/20 y también en los porcentajes respecto a los cursos anteriores.

El porcentaje de aprobados en este bloque de contenidos de la asignatura al completar la convocatoria ordinaria alcanzó un valor ligeramente superior al $90 \%$, con una calificación superior a 7, siendo ambos valores de los más altos en los cursos analizados. Esto implica que el material didáctico ha conseguido también que un mayor porcentaje de estudiantes 
tenga un seguimiento regular de esta parte de la asignatura pudiendo superarla en la primera convocatoria oficial de evaluación, tabla 2.

Tabla 1. Resumen de resultados académicos prueba parcial.

\begin{tabular}{ccccccc}
\hline \multicolumn{5}{c}{ Test de conceptos } \\
\hline Curso & $15 / 16$ & $16 / 17$ & $17 / 18$ & $18 / 19$ & $19 / 20$ & $20 / 21$ \\
Estudiantes & 51 & 53 & 62 & 49 & 49 & 40 \\
Aprobados & 16 & 17 & 11 & 24 & 29 & 18 \\
\% & 31,4 & 32,1 & 17,7 & 49,0 & 59,2 & 45,0 \\
Calif. media & 6,71 & 6,44 & 6,43 & 6,86 & 6,03 & 7,25 \\
Calif. $>4$ & 31 & 29 & 20 & 29 & 42 & 30 \\
$\%$ & 60,8 & 54,7 & 32,3 & 59,2 & 89,8 & 75,0 \\
\hline \% & & & Problema & & \\
\hline Curso & $15 / 16$ & $16 / 17$ & $17 / 18$ & $18 / 19$ & $19 / 20$ & $20 / 21$ \\
Estudiantes & 51 & 53 & 62 & 44 & 46 & 38 \\
Aprobados & 13 & 12 & 13 & 12 & 29 & 19 \\
\% & 25,5 & 22,6 & 21,0 & 27,3 & 63,0 & 50,0 \\
Calif. media & 7,56 & 5,61 & 6,15 & 8,33 & 7,43 & 8,58 \\
Calif. $>4$ & 13 & 14 & 14 & 17 & 35 & 20 \\
\% & 25,5 & 26,4 & 22,6 & 38,6 & 76,0 & 52,6 \\
\hline
\end{tabular}

Tabla 2. Resumen de resultados académicos finales.

\begin{tabular}{lcccccc}
\hline & \multicolumn{6}{c}{$\begin{array}{c}\text { Resultados finales del bloque de contenidos la } \\
\text { finalizar la convocatoria ordinaria }\end{array}$} \\
\hline Curso & $15 / 16$ & $16 / 17$ & $17 / 18$ & $18 / 19$ & $19 / 20$ & $20 / 21$ \\
Presentados & 53 & 51 & 54 & 51 & 50 & 41 \\
Aprobados & 29 & 39 & 25 & 28 & 43 & 37 \\
$\quad \%$ & 54,7 & 76,5 & 46,3 & 54,9 & 86,0 & 90,2 \\
$\begin{array}{c}\text { Calificación } \\
\text { media }\end{array}$ & 7,10 & 6,69 & 6,68 & 5,85 & 6,29 & 7,04 \\
\hline
\end{tabular}

El equipo docente responsable de evaluar este bloque de contenidos durante los cursos académicos estudiados ha sido el mismo, y ha mantenido unos criterios de evaluación homogéneos que permiten realizar una comparación fiable de estos resultados. Se ha observado una significativa mejora en el nivel de dominio de los contenidos, y en la calidad de los argumentos justificativos de las soluciones aportadas en el problema de aplicación práctica.

A partir de toda esta información, se puede inferir que la introducción de este nuevo material ha tenido un impacto positivo en los resultados académicos y con un nivel de satisfacción alto entre los estudiantes. Ellos también han destacado en sus valoraciones de forma muy positiva el trabajo realizado por un compañero de la titulación en su trabajo fin de grado, para la mejora y adaptación de este material didáctico a la plataforma Moodle.

\section{CONCLUSIONES}

La participación activa y comprometida de estudiantes en la mejora del proceso de enseñanza y aprendizaje se ha mostrado muy positiva en esta experiencia.

Se ha evidenciado que la mejora en el grado de adaptatividad de este material didáctico ha supuesto que un alto porcentaje de estudiantes se sintieran motivados para hacer un seguimiento regular de los contenidos del mismo y ser capaces de superarlos en las primeras pruebas de evaluación.

Algunos estudiantes han solicitado que sería interesante que se pudieran descargar los contenidos del material didáctico para poder usarlo sin conexión a internet. Se considera que esta opción no sería conveniente pues se pierde la adaptatividad y progresividad aportada en la presentación de los contenidos y ejerciendo un efecto desincentivador para el grupo en su conjunto.

El material didáctico de partida ya se viene usando también de forma exitosa en asignaturas equivalentes de otros grados de ingeniería, por lo que esta positiva experiencia se considera totalmente transferible, e incluso con un impacto potencial más elevado en asignaturas de menor número de créditos.

Muchos de los estudiantes que no superaron total o parcialmente estos contenidos en la prueba de evaluación parcial, han podido finalmente superarla en la convocatoria ordinaria sin necesidad de tutorías adicionales. Se considera que esto es un indicador de una alta eficacia del material didáctico en su utilización de forma autónoma cuando el estudiante muestra un elevado interés en su uso.

En cursos anteriores con docencia presencial el material didáctico se ha usado en una sesión de teoría para su introducción durante unos 30 minutos y en dos sesiones más de prácticas de aula de 2 horas, dirigidas a la aplicación práctica en ejercicios y la resolución de problemas, como recurso de apoyo. Al inicio de la primera sesión de prácticas de aula el estudiante tiene que realizar un cuestionario evaluable, cuya finalidad es lograr que hayan adquirido al menos las nociones básicas imprescindibles para seguir estas sesiones. En este curso académico 20/21 el nuevo material didáctico se ha tenido que usar de forma telepresencial y se ha empleado el mismo tiempo y el mismo número de sesiones. A pesar de no contar con la interacción más rápida y directa que se lograba en las sesiones presenciales, el contar con el material didáctico integrado en la plataforma Moodle ha permitido la participación activa de los estudiantes que interactuaban con los diferentes recursos mientras se realizaba la resolución de los ejercicios y problemas.

Los estudiantes han indicado que se requiere un media de entre 6 y 8 horas para la primera visualización del conjunto de contenidos y una dedicación media similar para el estudio previo a la prueba de evaluación. En un trabajo previo se estimó el tiempo de dedicación del estudiante, con el material didáctico de partida para superar la asignatura, y su valor fue en torno a un $20 \%$ más alto. Habría que analizar con más detalle este aspecto que parece indicar, junto con los mejores resultados académicos obtenidos, una mayor eficiencia del tiempo dedicado por el estudiante con esta nueva versión del material didáctico.

Un aspecto de mejora, sugerido por los estudiantes, sería la introducción de cuestionarios de autoevaluación específicos 
para cada bloque, donde pudieran realizar un mayor número de intentos de prueba antes de acometer los cuestionarios que dan acceso al resto de contenidos. Con una adecuada configuración del banco de preguntas para ambos tipos de cuestionarios sería relativamente sencillo adaptar la utilización del material didáctico en asignaturas con una menor carga de créditos y que no requieran de un nivel de profundidad alto en este bloque de contenidos. Esta flexibilidad de uso permite que esta experiencia sea transferible a otros títulos de grados en ingenierías.

\section{AgRADECIMIENTOS}

Se quiere agradecer especialmente a los estudiantes que han cursado la asignatura de Procesos Industriales del Grado en Ingeniería en Diseño Industrial y Desarrollo de Productos por su participación y colaboración en esta experiencia.

\section{REFERENCIAS}

Becker, S. A., Becker, S. A., Cummins, M., Davis, A., Freeman, A., Hall, C. G., \& Ananthanarayanan, V. (2017). NMC Horizon Report: 2017 Higher Education Edition.

Bovill, C., \& Felten, P. (2016). Cultivating student-staff partnerships through research and practice. In International Journal for Academic Development (Vol. 21, Issue 1, pp. 1-3). Routledge. https://doi.org/10.1080/1360144X.2016.1124965

Cronin, C., \& Maclaren, I. (2018). Conceptualising OEP: A review of theoretical and empirical literature in Open Educational Practices. Open Praxis, 10(2), 127-143. https://doi.org/10.5944/openpraxis.10.2.825

EduTrends. (2014). Aprendizaje Adaptativo. In Observatorio de Innovación Educativa. https://observatorio.itesm.mx/edutrendsaprendizajeadapt ativo/

Enfield, J. (2016). The Value of Using an E-Text in a Flipped Course. TechTrends, 60(5), 449-455. https://doi.org/10.1007/s11528-016-0100-1

García-Peñalvo, F. J., Fidalgo-Blanco, Á., Sein-Echaluce, M. L., \& Sánchez-Canales, M. (2019). Active Peer-Based Flip Teaching (pp. 1-16). https://doi.org/10.4018/978-15225-8142-0.ch001

Graduate XXI. (2018). Las 10 plataformas de aprendizaje adaptativo que viajan al futuro de la educación. In
Graduate XXI. http://www.graduatexxi.org/las-10plataformas-de-aprendizaje-adaptativo-que-viajan-alfuturo-de-la-educacion/

Hernández-Castellano Pedro M., Marrero-Alemán, M. D., Paz-Hernández, R., Bordón-Pérez, P., Suárez-García, A., \& Luis. (2017). Impacto de la introducción de un material didáctico interactivo en los resultados de aprendizaje en asignaturas de grado de ingenierías. Actas Del IV Congreso Internacional Sobre Aprendizaje, Innovación y Competitividad, CINAIC 2017. https://doi.org/10.26754/CINAIC.2017.000001_025

Hernández-Castellano, Pedro M, Marrero-Alemán, M. D., Aranda-Loureiro, A. M., Ortega-García, F., PazHernández, R., \& Benítez-Vega, A. N. (2017). Development of Interactive Learning Materials in Engineering of Manufacturing Processes. Materials Science Forum, 903, 63-69. https://doi.org/10.4028/www.scientific.net/MSF.903.63

Hernández-Castellano, Pedro Manuel, Marrero-Alemán, M. D., Paz-Hernández, R., Bordón-Pérez, P. R., SuárezGarcía, L. A., \& Benítez-Vega, A. N. (2019). Adaptive Learning Using Interactive Training Material. Innovative Trends in Flipped Teaching and Adaptive Learning, 162-184. https://doi.org/10.4018/978-1-52258142-0.ch008

Lerís, D., \& Sein-Echaluce, M. L. (2011). La personalización del aprendizaje: un objetivo del paradigma educativo centrado en el aprendizaje. Arbor, 187(Extra_3), 123134. https://doi.org/10.3989/arbor.2011.Extra-3n3135

López, D. L., Muniesa, F. V., \& Gimeno, Á. V. (2015). Aprendizaje adaptativo en moodle: tres casos prácticos/Adaptive learning in moodle: three practical cases. Education in the Knowledge Society; Salamanca, 16(4), 138-157.

https://doi.org/http://dx.doi.org.bibproxy.ulpgc.es/10.14 201/eks201516138157

Matthews, K. E., Cook-Sather, A., Acai, A., Dvorakova, S. L., Felten, P., Marquis, E., \& Mercer-Mapstone, L. (2019). Toward theories of partnership praxis: an analysis of interpretive framing in literature on students as partners in teaching and learning. Higher Education Research and Development, 38(2), 280-293. https://doi.org/10.1080/07294360.2018.1530199 